\title{
The Effects of Nitroglycerin on Coronary Collaterals and Myocardial Contractility
}

\author{
Michael V. Cohen, James M. Downey, Edmund H. Sonnenblick, and \\ EDWARD S. KIRK \\ From the Cardiovascular Division, Department of Medicine, Peter Bent \\ Brigham Hospital and Harvard Medical School, \\ Boston, Massachusetts 02115
}

\begin{abstract}
A B S T R A C T Nitroglycerin (TNG) causes a prolonged dilatation of coronary collaterals. To demonstrate a functional significance of this dilatation we measured the effect of TNG on myocardial contractile force in dogs $2 \frac{1}{2}-4$ wk after the left anterior descending coronary artery (LAD) had been embolized in closed-chest animals. Development of collaterals was documented by angiography. Via a left thoracotomy the main left coronary artery (LCA) and LAD distal to the embolized plug were cannulated. Coronary flow and perfusion pressure were recorded. Contractile force was measured with gauges sutured to epicardial areas supplied by the left circumflex coronary artery (LCf) and occluded LAD. Coronary perfusion pressure in the LCA was gradually decreased until the contractile force recorded by the LAD gauge diminished while the LCf gauge was unaffected. Under these conditions, with coronary perfusion pressure held constant with the aid of a Starling resistance, TNG $(18 \mu \mathrm{g})$ injected into the LCA increased peripheral LAD pressure by $3-12 \mathrm{~mm} \mathrm{Hg}$ and contractile force in the LAD region by $36 \%$ (range $20-90 \%$ ), returning it to near-normal levels, while having minimal effect in the LCf area. These changes persisted for 5 min. When LCf and LAD areas were both ischemic, intracoronary TNG had minimal effect on peripheral LAD pressure and contractile force. Thus, TNG causes prolonged dilatation of coronary collaterals and presumed increased collateral flow with subsequent enhancement of myocardial contractile force in ischemic areas. This effect is seen only when ischemia is limited to an area supplied by the collaterals. When the whole heart is
\end{abstract}

This study was presented in part at the 22nd Annual Scientific Session of the American College of Cardiology, San Francisco, California, February 1973. An abstract appeared in Am. J Cardiol. $31: 126$.

Received for publication 5 February 1973 and in revised form 2 July 1973. ischemic, collaterals are unresponsive to TNG, suggesting that these collaterals dilate fully when the regions from which they originate become ischemic.

\section{INTRODUCTION}

Although nitroglycerin ( $T N G)^{1}$ has been used for more than a century for the relief of angina pectoris, its mode of action remains uncertain. There is little doubt that nitrites decrease coronary vascular resistance $(1,2)$ and augment coronary flow in normal man (3-5). Furthermore, relaxation of coronary vascular smooth muscle has been documented in helical strips of coronary arteries (6), models in intact dogs (7), and even coronary arteries of man (7). Despite these observations, the clinical efficacy of TNG has most often been attributed to its peripheral effects (8) resulting in diminished myocardial oxygen consumption (9) and restoration of oxygen balance in ischemic myocardium. The ability of TNG to increase flow in atherosclerotic vessels has been questioned. However, with refined techniques investigators have documented an early increase in coronary blood flow in patients with abnormal vessels that is later obscured by the peripheral hypotensive effect of TNG $(5,10)$. A biphasic effect of TNG on coronary blood flow is supported by carefully controlled studies in animals (11-13). Moreover, a direct dilatory effect of TNG on coronary collaterals in dog (14) and man (15) has been shown, although the significance of the direct coronary effects of TNG is undermined by the recent report that only intravenous and not intracoronary administration of TNG is effective in the relief of angina in man (5). To help de-

\footnotetext{
${ }^{1}$ Abbreviations used in this paper: LAD, left anterior descending coronary artery; LCA, main left coronary artery; LCf, left circumflex coronary artery; PCP, peripheral LAD pressure; TNG, nitroglycerin.
} 
fine the direct coronary effects of $\mathrm{TNG}$, we have reexamined the effect of this drug on coronary collateral vessels in a chronically ischemic canine model. The investigation reported here documents the ability of $T N G$ to dilate collateral vessels and significantly improve contractility in ischemic myocardium, presumably by enhancing blood flow to the collateralized ischemic areas.

\section{METHODS}

Experiments were performed on 11 mongrel dogs. Three dogs were used in acute experiments immediately after ligation of the left anterior descending coronary artery (LAD). By a closed-chest technique described elsewhere (16), a gradual occlusion of the LAD was produced $2 \frac{1}{2}-4$ wk before the time of study in eight dogs. Briefly, a 3-5 $\mathrm{mm}$ segment of red $\mathrm{Kifa}^{2}$ catheter (6.6 French) was embolized into the LAD with a coaxial catheter system introduced into a femoral artery. The catheter system consisted of an outer red Kifa catheter and an inner Teflon catheter extending beyond the end of the former and carrying a hollow, tapered Kifa plug. Once the catneter system was maneuvered into the $\mathrm{LAD}$, the Kifa catheter was anchored while the inner catheter was withdrawn into the outer one, thus releasing the plug into the LAD. 1-3 days before the acute study all dogs had coronary angiograms demonstrating complete occlusion of the LAD at the level of the plug with retrograde filling of the distal vessel from numerous collaterals. All dogs had careful visual inspection of the infarcted area at the termination of the study. The hearts were serially sectioned from apex to base and the areas of necrosis and of normal myocardium were measured by planimetry from photographs of the sections. In the group of eight an average of $4 \pm 2 \%$ of the left ventricular cross-sectional area was seen to have been infarcted. Four of these had no detectable infarct.

On the day of study the dogs were anesthetized with pentobarbital $(27.5 \mathrm{mg} / \mathrm{kg})$. Additional amounts were administered as required to maintain prolonged anesthesia. A polyvinyl catheter was introduced into the thoracic aorta via a femoral artery for measurement of systemic pressure with a Statham transducer (Statham Instruments, Inc. Oxnard, Calif.). After endotracheal intubation and mechanical ventilation with an intermittent positive pressure respirator with $100 \%$ oxygen, a left thoracotomy was performed. Anticoagulation was produced by injection of 20,000 $\mathrm{U}$ of heparin and maintained by injection of $10,000 \mathrm{U}$ every $1-2 \mathrm{~h}$.

The experimental preparation is diagrammed in Fig. 1. The main left coronary artery (LCA) was cannulated with a modified Gregg cannula introduced into the left subclavian artery. Blood from the root of the aorta entered a side hole in the double-lumen shaft of the cannula, passed through a loop, and then returned via the other lumen to the coronary artery. The loop contained an extracorporeal flow probe attached to a Biotronex 410 flowmeter (Biotronex Laboratory, Inc., Silver Spring, Md.), a side arm for measurement of coronary perfusion pressure with a Statham transducer, and an area for intracoronary drug injection. These preparations showed marked pressure-independent autoregulation of coronary blood flow : steady-state coronary resistance decreased when perfusion pressure was lowered from the control level (17). Perfusion at constant pressure

${ }^{2}$ United States Catheter \& Instrument Corp., Glens Falls, N. Y. was achieved with the aid of a Sigmamotor pump (Sigmamotor, Inc., Middleport, N. Y.) and a Starling resistance (Fig. 1). Flow through the pump was increased until the flow occurred continuously through the Starling resistance. The level of perfusion pressure was then that pressure at which this overflow occurred. Perfusion pressure was easily varied by adjusting the pressure within the jacket of the Starling resistance. The LAD was isolated just distal to the embolized plug and cannulated with a 20-gauge needle for measurement of peripheral LAD pressure (PCP) with a Statham transducer.

Myocardial contractile force was measured with isometric strain gauge arches. ${ }^{3}$ One arch was sewn to the epicardium in the area supplied by the left circumflex coronary artery (LCf) in an orientation parallel to the superficial fibers. The area supplied by the LAD was demarcated by injecting saline down the LAD cannula and noting the boundary of the blanched area. A second strain gauge arch was sewn onto the surface in this area in an orientation parallel to the underlying epicardial fibers. The responsiveness of the gauges to total coronary occlusion was noted. If either gauge was unresponsive or passively followed changes in ventricular pressure, the position of the gauge was altered. Isoproterenol was flushed down the LAD cannula, and the selective vigorous response of the LAD gauge documented that it recorded changes in contractility of the area supplied by the occluded LAD. All signals were recorded directly on a multichannel oscillograph.

Our experimental protocol used isometric strain gauge arches to monitor the myocardial effects of decreased coronary perfusion pressure and administered drugs. The strain gauge arch records forces dependent on local metabolic processes. Sequential falls in coronary flow are associated with progressive loss of amplitude of strain gauge arch deflections. Thus the gauges enabled us to assess the extent of myocardial ischemia. The increase in amplitude of the strain gauge arch recording with the resumption of a higher coronary flow indicates a flow-dependent state of the myocardium underlying the gauge and serves as our operational definition of ischemia. This qualitative approach avoids the difficulty of defining ischemia by setting an arbitrary level of tissue metabolites or electrical events. To determine the effect of drugs on ischemic myocardium, we documented the changes in the deflection of the strain gauge arch after the intracoronary administration of these agents. The intracoronary route minimizes potential systemic hemodynamic effects. In preliminary experiments, careful measurement of aortic and ventricular pressures showed that the selected drug doses did not alter ventricular preload or afterload. Therefore, drug-induced alterations of strain gauge arch recordings reflect changes in contractile force. Myocardial blood flow measurements can indicate only flow changes mediated by drugs, and cannot indicate the functional significance of a given change in flow to ischemic myocardium. Only functional measurements can indicate this significance. Furthermore, a recent study from this laboratory (18) has demonstrated that strain gauge arches are sensitive indicators of myocardial blood flow when contractile force is diminished in ischemic situations. Increased contractility of the small ischemic area was not expected to significantly enhance left ventricular performance, and therefore changes in ventricular pressure were not consistently monitored.

Contractile force responses to TNG could not be mea-

${ }^{3}$ John Warren, Charlestown, S. C. 


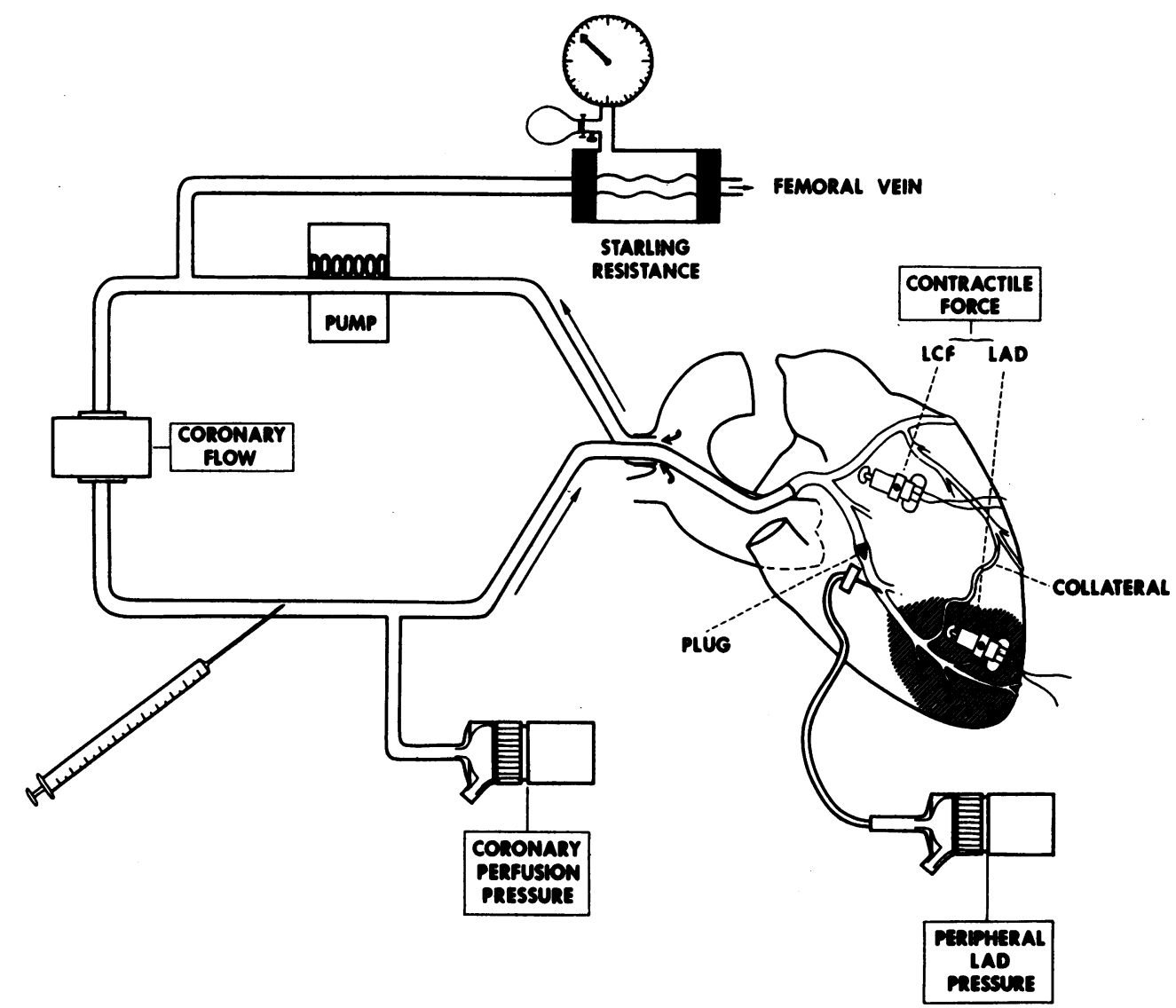

Figure 1 Experimental preparation. The shading of the left ventricle denotes the area supplied by collateral vessels.

sured in two animals. One dog had a large infarct $(16 \%$ of the left ventricular cross-sectional area). Consequently it was not possible to find enough viable yet ischemic myocardium from which to record changes in contractile force. One additional animal had a very small gradient from LCA to distal LAD ( $9 \mathrm{~mm} \mathrm{Hg}$ ), which made it impossible to cause the LAD region to be selectively ischemic by restricting LCA inflow (see below).

Three dogs with intact coronary circulations at the time of study were used as controls. The preparation was identical to that above except that the LAD was acutely ligated in its middle third and then cannulated distal to the ligature.

All drugs were given into the isolated coronary artery in doses that were too small to cause systemic effects. A solution of TNG was prepared by dissolving $0.6-\mathrm{mg}$ tablets (Eli Lilly \& Co., Indianapolis, Ind.) in saline to make a final concentration of $0.6 \mu \mathrm{g} / \mu \mathrm{l}$. The standard intracoronary dose was $18 \mu \mathrm{g}$. The dose was chosen to be one with a maximal dilating effect. It approximates the amount delivered in the first circulation to the coronary arteries after absorption of the usual sublingual therapeutic dose in man. Since the effect of TNG on collaterals persists for several minutes (see below), the route of administration may not be critical. Adenosine (Sigma Chemical Co., Inc. St. Louis, Mo.) solution was prepared just before its use each time. The final concentration was $1 \mu \mathrm{g} / \mu \mathrm{l}$ in saline and the intracoronary dose used was $30 \mu \mathrm{g}$.
In three chronically prepared animals the response of the LAD and LCf strain gauge arches to varying coronary perfusion was examined by gradually increasing and then decreasing the coronary flow with the Sigmamotor pump in steps of $10-15 \mathrm{ml} / \mathrm{min}$. In all animals perfusion pressure was then gradually decreased in a stepwise fashion and the Starling resistance used to maintain a constant perfusion pressure. At each level TNG was injected into the LCA and the response of strain gauge arches, PCP, and coronary flow were recorded. Adenosine was similarly tested in three animals for its effect on contractile force and PCP.

\section{RESULTS}

Acute LAD occlusion. At the time of cannulation of the distal LAD, peripheral LAD pressure was less than $35 \mathrm{~mm} \mathrm{Hg}$. The gradient from LCA to distal LAD was $70 \mathrm{~mm} \mathrm{Hg}$. After the administration of intracoronary TNG, coronary flow increased while the perfusion pressure was minimally and only transiently depressed. Pressure in the distal portion of the LAD was not altered. Because of the severe ischemia caused by acute occlusion of the LAD, myocardial contraction in the area ceased, and the LAD strain gauge arch became essentially unresponsive and an unreliable index of the effect of intracoronary TNG. 


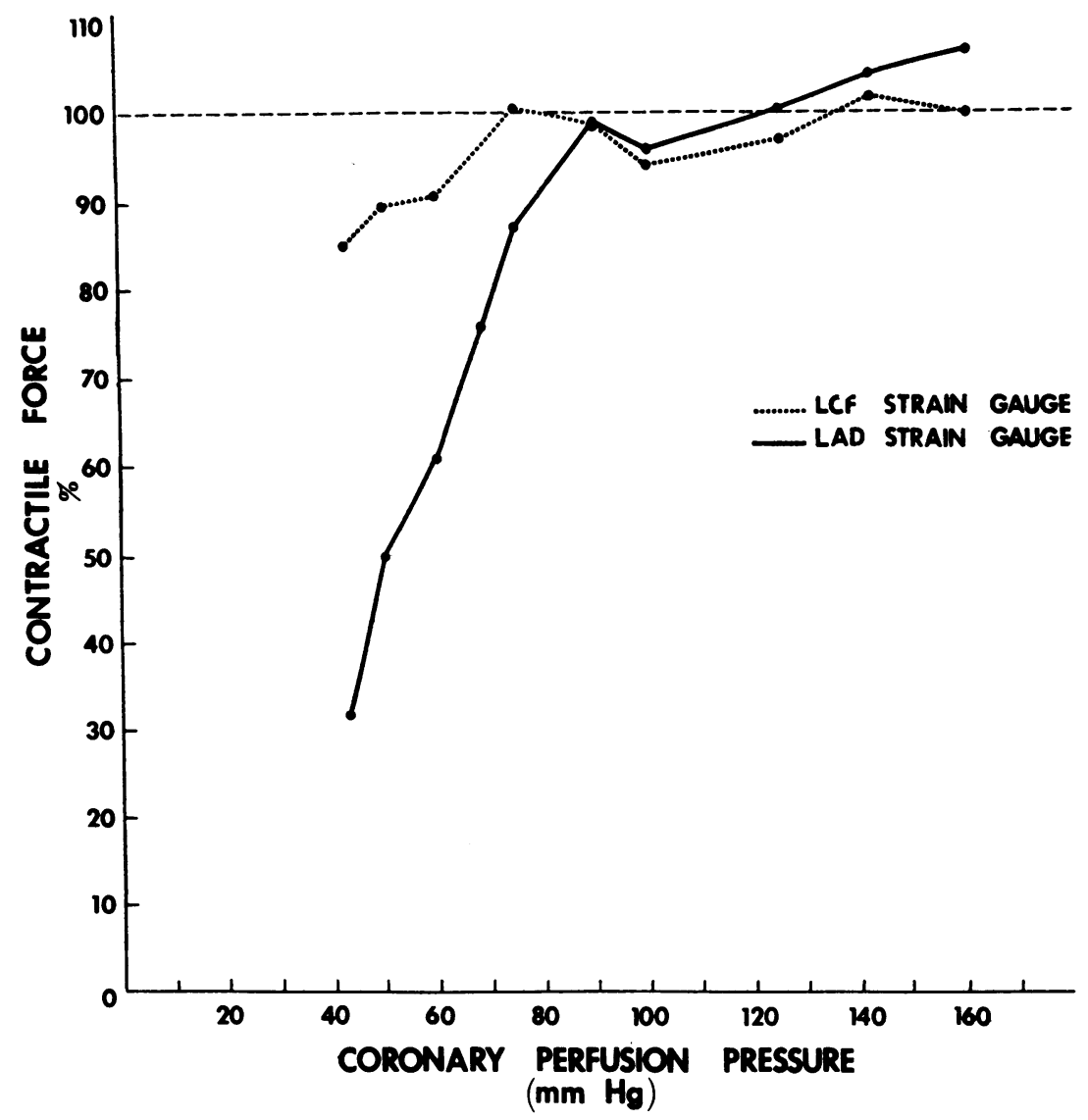

FigURE 2 Effect of increased and decreased mean perfusion pressure in the main LCA on the response of the strain gauge arches in the normal area (LCf) and area distal to the occluded LAD. By lowering coronary perfusion it was possible to produce a marked differential ischemia.

Chronic LAD occlusion. Under control conditions, pressure in the peripheral LAD ranged from $47-100 \mathrm{~mm}$ $\mathrm{Hg}$ (average $63 \mathrm{~mm} \mathrm{Hg}$ ), while the average gradient between the LCA and distal LAD was $36 \mathrm{~mm} \mathrm{Hg}$. Coronary flow in the control state averaged $75 \mathrm{ml} / \mathrm{min}$. The effect of varying coronary perfusion on the response of the strain gauge arches is shown in Fig. 2. The LCf gauge was relatively uninfluenced by changing perfusion pressure until pressures below $75 \mathrm{~mm} \mathrm{Hg}$ were achieved. Below this level the response of the gauge declined slowly and at a perfusion pressure of $43 \mathrm{~mm} \mathrm{Hg}$ decreased by $15 \%$. This response, however, is contrasted with that of the gauge in the ischemic area. The response was similarly unvarying during supernormal pressures and flows, but at pressures below the control level of $90 \mathrm{~mm} \mathrm{Hg}$ the gauge response fell precipitously and at the lowest perfusion pressure of $43 \mathrm{~mm} \mathrm{Hg}$ was markedly depressed to $32 \%$ of control. Although the absolute changes varied between animals, the responses were qualitatively similar. Therefore, by lowering coronary perfusion pressure it was possible to make the area of the LAD quite ischemic while preserving the contractile force in the region of the LCf.

To determine the effects of TNG, coronary perfusion pressure in the main LCA was decreased until there was a differential effect on the strain gauges. In Fig. 3 the responses from one representative experiment are shown. In the control state coronary perfusion pressure was 92 $\mathrm{mm} \mathrm{Hg}$ and the gradient between the LCA and distal $\mathrm{LAD}$ was $45 \mathrm{~mm} \mathrm{Hg}$. Before administration of TNG the perfusion pressure was decreased to $51 \mathrm{~mm} \mathrm{Hg}$, leaving a gradient of $35 \mathrm{~mm} \mathrm{Hg}$. At this level of perfusion the LAD area clearly was ischemic, as shown by the marked fall in the contractile force. In contrast the contractile force in the nonischemic LCf area was essentially unchanged. Intracoronary administration of TNG $(18 \mu \mathrm{g})$ increased coronary blood flow. PCP rose by $10 \mathrm{~mm} \mathrm{Hg}$, and the pressure gradient between the LCA and peripheral LAD decreased to $25 \mathrm{~mm} \mathrm{Hg}$ despite a constant coronary perfusion pressure. Concomitantly, the con- 


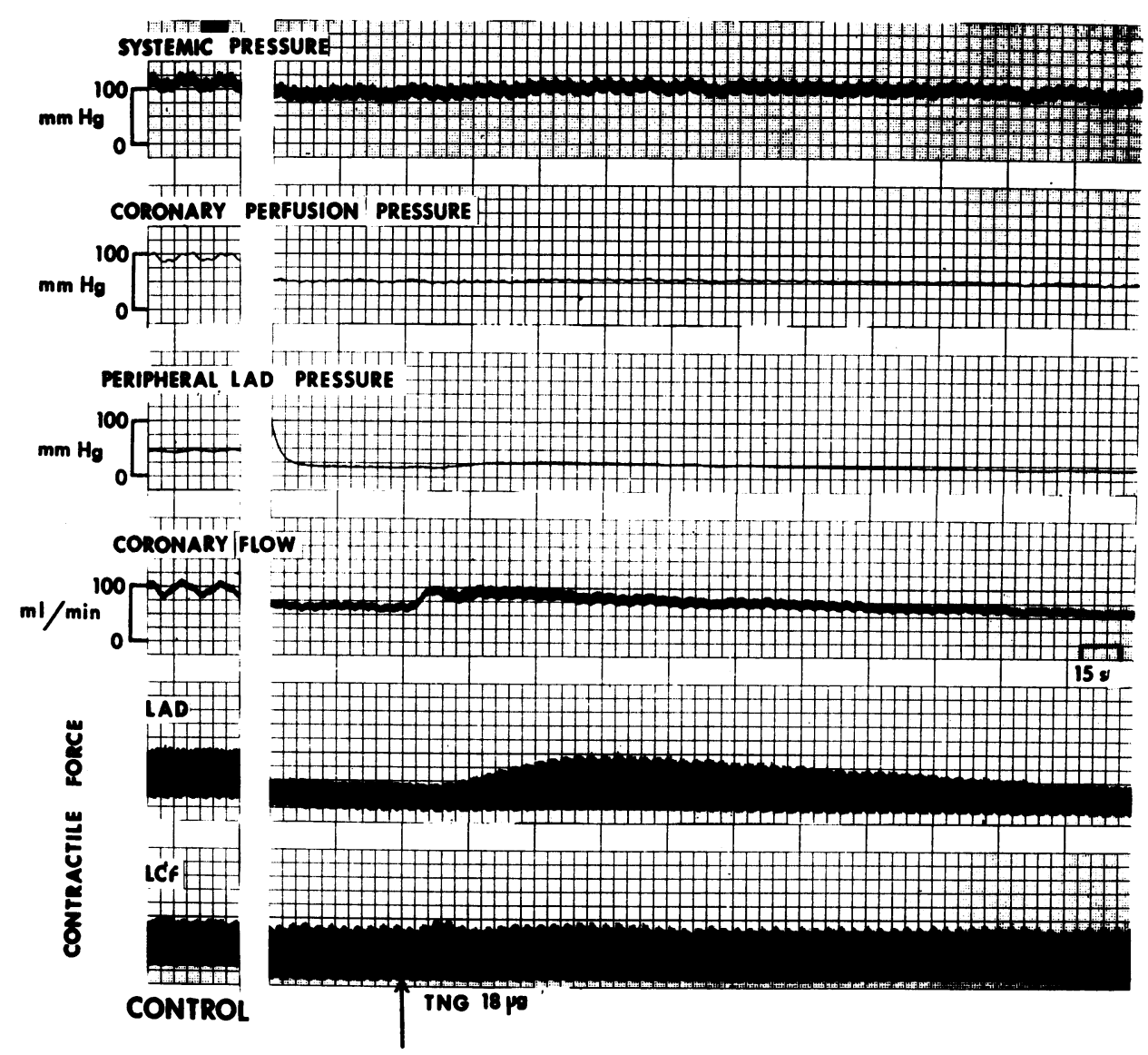

FIGURE 3 Effect of intracoronary TNG on the peripheral LAD pressure and contractile force in the LAD and LCf areas. Before TNG administration the coronary perfusion pressure had been lowered from the control level of $92 \mathrm{~mm} \mathrm{Hg}$ to $51 \mathrm{~mm} \mathrm{Hg}$. At this perfusion pressure TNG elicited increases in PCP and LAD contractile force, but did not significantly alter LCf contractile force.

tractile force in the LAD area increased markedly by $90 \%$ of the pre-TNG level while the LCf strain gauge arch was not affected.

For the six animals studied, the effect of TNG was maximal when the initial coronary perfusion pressure was lowered by an average of $40 \mathrm{~mm} \mathrm{Hg}$. At this pressure the LCf strain gauge arch was depressed only $4 \pm 1 \%$ (SEM) whereas the LAD gauge was depressed by $29 \pm 4 \%$ (SEM) of control (Fig. 4). This difference is highly significant $(P<0.001)$. The maximal enhancement of the contractile force developed by the myocardium in the LAD area compared to the stable level just before administration of TNG averaged 36\% (range $20-90 \%)$. However, the LCf gauge barely responded (average increase $2 \%$, range $0-4 \%$ ). When the contractile force at the peak of TNG's effect is compared to the measurement at normal perfusion pressure, it is evident that TNG can transiently restore the contractile force in the ischemic LAD region to near-normal levels (average
$4 \%$ below control). TNG always increased pressure in the LAD distal to the occlusion (average $7 \mathrm{~mm} \mathrm{Hg}$, range $3-12 \mathrm{~mm} \mathrm{Hg}$ ). The rise was gradual and the effect persisted for $2 \frac{1}{2}-8 \mathrm{~min}$ (average $5 \mathrm{~min}$ ).

The response to TNG was shown to be highly dependent on the level of the coronary perfusion pressure. A representative record of this is reproduced in Fig. 5 and results for all animals are tabulated in Table I. At the control coronary perfusion pressure of $97 \mathrm{~mm} \mathrm{Hg}$ (left panel, Fig. 5), neither the LAD nor LCf region was ischemic as indicated by failure of contractile force to increase when perfusion pressure was raised (see Fig. 2). Although intracoronary TNG raised PCP by $4 \mathrm{~mm} \mathrm{Hg}$ and increased coronary blood flow, myocardial contractile force was not altered. However, at a perfusion pressure of $75 \mathrm{~mm} \mathrm{Hg}$ (middle panel, Fig. 5), the LAD area was ischemic, as indicated by a $30 \%$ decrease in contractile force, whereas the LCf gauge showed only a $5 \%$ decrease. Under these conditions, intracoronary TNG 
elicited an increase in PCP of $6 \mathrm{~mm} \mathrm{Hg}$, augmented coronary flow, and enhanced contractile force in the LAD region. There was no change in contractile force in the area of the LCf. When the perfusion pressure was lowered further to $50 \mathrm{~mm} \mathrm{Hg}$ (right panel, Fig. 5), contractile force in the LAD region was decreased by an additional $10 \%$, but now the LCf gauge registered a $25 \%$ decrease compared to control. Under these conditions, intracoronary TNG produced only a minor increase in coronary blood flow and was ineffective in altering either PCP or myocardial contractile force.

In three animals intracoronary adenosine was administered and a representative record is reproduced (Fig. 6). As above, contractile force in the LAD region had been reduced. After injection of adenosine, coronary flow rose transiently while PCP increased an average of $5 \mathrm{~mm} \mathrm{Hg}$ (range $2-10 \mathrm{~mm} \mathrm{Hg}$ ). Increases in contractility were less pronounced than after TNG. The LAD strain gauge arch registered a $0-12 \%$ (average $6 \%$ ) increase, while the LCf gauge was unaffected. The measured parameters remained above control levels for $3 \frac{1}{2}-4 \mathrm{~min}$.

\section{DISCUSSION}

This study has demonstrated a direct action of TNG on the coronary vasculature to dilate coronary collaterals and subsequently enhance the contractility of ischemic myocardium presumably by augmenting collateral flow.

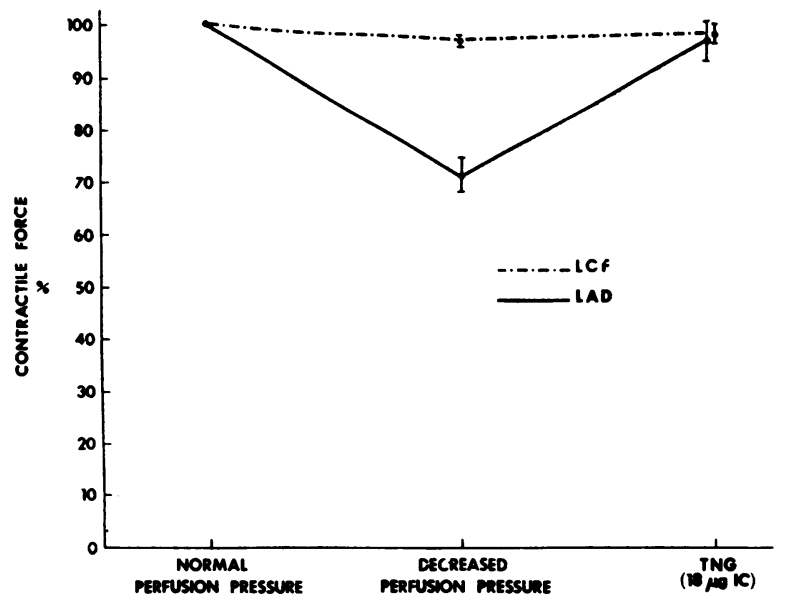

FIGURE 4 Summary graph illustrating enhancement of myocardial contractile force by the effect of TNG on coronary collaterals. Before TNG administration perfusion pressure was decreased. This induced ischemia in the area supplied by collaterals (LAD) as evidenced by the fall in contractile force. Intracoronary (IC) TNG transiently restored the contractile force to a near-normal level. The changes in the normal (LCf) area were insignificant. Vertical bars denote twice the SEM.

Our data confirm and expand the conclusions of Fam and McGregor (14), based on animal experiments, and the more recent results of Goldstein, Stinson, and Epstein

TABLE I

Effect of TNG on Peripheral LAD Pressure and Contractile Force at Different Levels of Coronary Perfusion Pressure

\begin{tabular}{|c|c|c|c|c|c|c|c|c|c|c|}
\hline \multirow[b]{2}{*}{$\begin{array}{c}\text { Dog } \\
\text { number }\end{array}$} & \multirow[b]{2}{*}{ State } & \multicolumn{3}{|c|}{ Control } & \multicolumn{3}{|c|}{ LAD Ischemia } & \multicolumn{3}{|c|}{ Global Ischemia } \\
\hline & & $\begin{array}{c}\Delta \text { LAD } \\
\text { contractile } \\
\text { force }\end{array}$ & $\begin{array}{c}\Delta \mathrm{LCf} \\
\text { contractile } \\
\text { force }\end{array}$ & $\begin{array}{l}\text { Peripheral } \\
\text { LAD } \\
\text { pressure }\end{array}$ & $\begin{array}{c}\Delta \text { LAD } \\
\text { contractile } \\
\text { force }\end{array}$ & $\begin{array}{c}\Delta \mathrm{LCf} \\
\text { contractile } \\
\text { force }\end{array}$ & $\begin{array}{l}\text { Peripheral } \\
\text { LAD } \\
\text { pressure }\end{array}$ & $\begin{array}{c}\Delta \text { LAD } \\
\text { contractile } \\
\text { force }\end{array}$ & $\begin{array}{c}\Delta \mathrm{LCf} \\
\text { contractile } \\
\text { force }\end{array}$ & $\begin{array}{c}\text { Peripheral } \\
\text { LAD } \\
\text { pressure }\end{array}$ \\
\hline \multirow{3}{*}{48} & & $\%$ & $\%$ & $m m \mathrm{Hg}$ & $\%$ & $\%$ & $m m \mathrm{Hg}$ & $\%$ & $\%$ & $m m \mathrm{Hg}$ \\
\hline & Pre-TNG & - & - & 47 & $-44 \ddagger$ & $0 \ddagger$ & 16 & - & - & - \\
\hline & Post-TNG* & $0 \ddagger$ & $+2 \ddagger$ & 62 & +5 & +2 & 26 & - & - & - \\
\hline \multirow[t]{2}{*}{342} & Pre-TNG & - & - & 53 & -30 & -5 & 32 & $-40 \ddagger$ & $-25 \ddagger$ & 27 \\
\hline & Post-TNG & +2 & 0 & 57 & -11 & -5 & 38 & -40 & -22 & 27 \\
\hline \multirow[t]{2}{*}{344} & Pre-TNG & - & - & 50 & -23 & -5 & 45 & -52 & -34 & 37 \\
\hline & Post-TNG & +1 & 0 & 59 & -5 & -5 & 57 & -52 & -34 & 37 \\
\hline \multirow[t]{2}{*}{419} & Pre-TNG & - & - & 70 & -37 & -3 & 35 & -50 & -20 & 25 \\
\hline & Post-TNG & 0 & +4 & 73 & -8 & -2 & 38 & -51 & -2.3 & 25 \\
\hline \multirow[t]{2}{*}{425} & Pre-TNG & - & - & 57 & -20 & -4 & 35 & -67 & -20 & 27 \\
\hline & Post-TNG & 0 & 0 & 60 & -4 & -3 & 42 & -68 & -20 & 27 \\
\hline \multirow[t]{2}{*}{781} & Pre-TNG & - & - & 100 & -22 & -4 & 50 & - & - & - \\
\hline & Post-TNG & +3 & +3 & 104 & +2 & 0 & 55 & - & - & - \\
\hline \multirow[t]{2}{*}{ Average } & Pre-TNG & 一** & -** & $63 \pm 8 \|$ & $-29 \pm 4$ I & $-4 \pm 1^{* *}$ & $36 \pm 59$ & $-52 \pm 6 * *$ & $-25 \pm 3^{* *}$ & $29 \pm 3^{* *}$ \\
\hline & Post-TNG & $+1 \pm 1 \S$ & $+2 \pm 1$ & $69 \pm 7$ & $-4 \pm 3$ & $-2 \pm 1$ & $43 \pm 5$ & $-53 \pm 6$ & $-25 \pm 3$ & $29 \pm 3$ \\
\hline
\end{tabular}

* All post-TNG measurements were made at the time of maximal response.

¥ All LAD and LCf strain gauge arch responses are related to the respective pre-TNG responses at control coronary perfusion pressure.

Statistical comparison (paired $t$ test) of pre- and post-TNG responses: Mean \pm SEM.

$\| P<0.025$

$\| P<0.005$.

** $P>0.05$. 

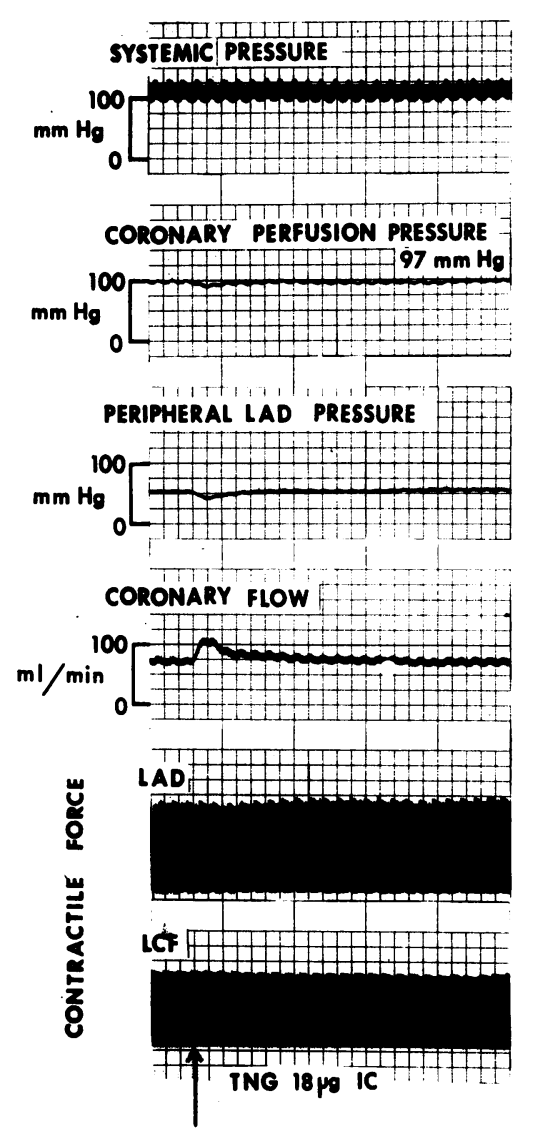
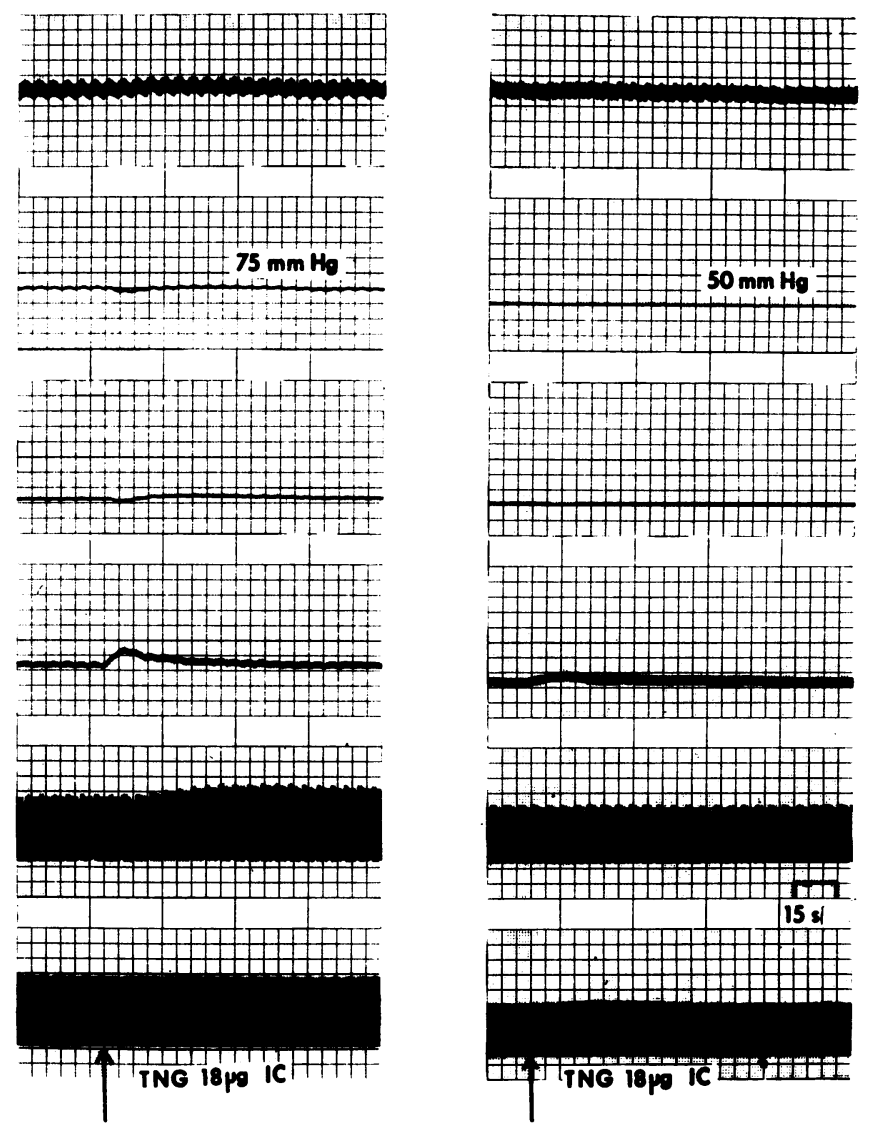

Figure 5 Effect of coronary perfusion pressure on the response of collaterals to intracoronary (IC) TNG. Whereas peripheral LAD pressure is increased after TNG administration at all but the lowest coronary perfusion pressures, LAD contractile force is augmented only at those pressures when both the myocardium is ischemic and coronary collaterals can be dilated, i.e., at intermediate perfusion pressures. Coronary flow increments after TNG administration that signify dilation of coronary arterioles become progressively less as perfusion pressure is lowered. With the initial increment of coronary flow, peripheral LAD pressure and contractile force transiently decrease, suggesting a coronary steal. However, arteriolar tone of the distal resistance vessels rapidly returns to control levels, signalling the end of the coronary steal and unmasking the longer time-course and effects of coronary collateral dilatation. In the middle panel a small rise in systemic pressure after TNG administration may reflect a transient increase in left ventricular contractility. However, this observation was not a consistent finding in these studies.

(19), obtained during intraoperative studies in man. With our experimental protocol, it is possible to make selected portions of the dog's left ventricular myocardium ischemic by varying coronary perfusion (Fig. 2). As demonstrated in Fig. 5, the ability of TNG to increase perfusion pressure in the distal portion of the obstructed LAD and thus augment myocardial contractile force is dependent on the level of coronary perfusion pressure. This observation forms the basis for our hypothesis of how TNG may act under these circumstances. The data are diagrammatically presented in Fig. 7. TNG appears to have a salutary effect on myocardial contractility only within a given range of perfusion pressures, from $\mathrm{A}$ to
B. At perfusion pressures corresponding to the left panel in Fig. 5 (pressure $>\mathrm{B}$ ), no area of the heart is ischemic. Under these conditions, TNG would not be expected to augment contractile force. Nevertheless, PCP is augmented by TNG. With a modest decrease of coronary perfusion pressure $(\mathrm{A} \leq$ pressure $\leq \mathrm{B})$, corresponding to the middle panel in Fig. 5, the area perfused by the LAD becomes ischemic, as witnessed by the decline in contractile force. TNG again increases PCP and flow, but in this situation also nearly restores the contractile force in the area of the LAD to the control level. As anticipated, the normal area of the LCf is unaffected. With a further decline in perfusion pressure 


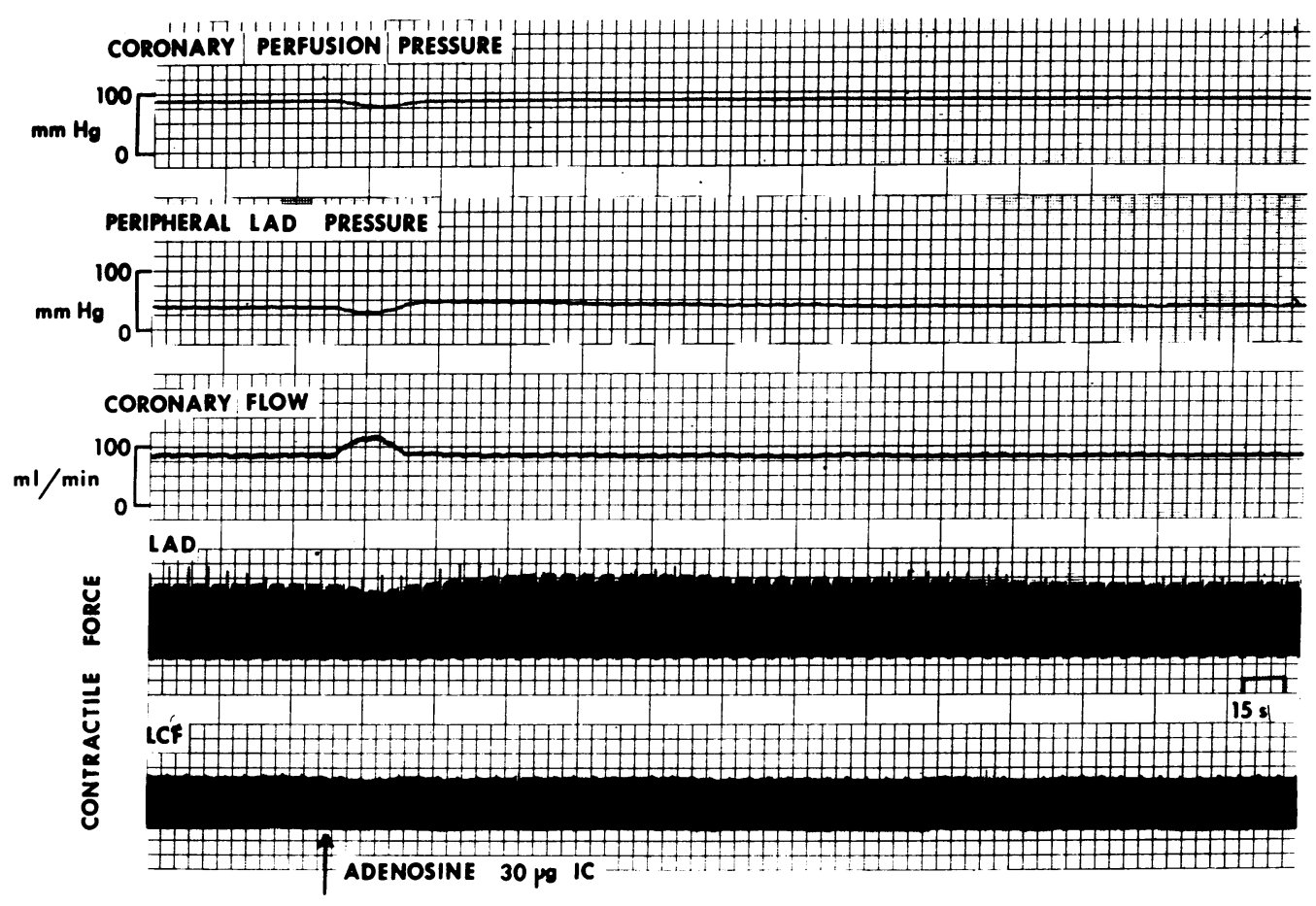

Figure 6 Effect of intracoronary (IC) adenosine on the peripheral LAD pressure and contractile force in the LAD and LCf areas. Experimental details are similar to those cited for Fig. 3.

(pressure $<A$ ), corresponding to the right panel in Fig. 5, both LAD and LC $f$ gauges indicate regional ischemia. Now TNG is ineffective in altering either PCP or contractile force.

Our model of the coronary collateral circulation relative to these findings is presented in Figs. 8 and 9. It is hypothesized that: $(a)$ the collateral vessel responds to metabolic stimuli, and $(b)$ the segment of a collateral ressel in an ischemic area dilates maximally in response to the hypoxic stimulus (Fig. 9). At a perfusion pressure where no portion of the myocardium is ischemic. intracoronary TNG dilates the collateral ressels but no myocardial effect is noted. The fall in resistance in the collateral vessel is documented by the observed increase in distal pressure in the occluded LAD. but there is no depressed myocardium to be improved by enhanced collateral flow. With the LAD area selectively ischemic, TNG dilates that portion of the collateral vessel in the area of the LCf. The enhanced collateral flow decreases the gradient of pressure across the collateral bed and thus improves the contractility of the depressed myocardium. With the initiation of global ischemia, all collateral and resistance vessels are maximally dilated, and now TNG can have no effect. The observation that the physiologic mediator adenosine (20) yields qualitatively similar results is consistent with this hypothesis.
If coronary collaterals are responsive to their metabolic milieu, the portion of the collateral vessel at the source cannot be responsive to the needs of the ischemic area. In our model TNG effectiveness is demonstrable only when the region supplying the collateral vessel is not ischemic, thus precluding maximal dilatation of the ressels. This may offer an explanation for the observation of Ganz and Marcus (5). If atrial pacing induced global as opposed to selective ischemia, one would not expect TNG to have a beneficial effect when delivered by the intracoronary route. However, the fact that coronary flow increased after intracoronary TNG in some patients suggests that not all of the myocardium was ischemic.

Our experimental protocol employed decreased coronary perfusion as the provocative stress to induce regional ischemia. This stress was particularly easy to initiate and adjust. I possible clinical analogue exists. The ischemic myocardium distal to a total coronary artery occlusion is supplied by collaterals. Should the source vessel to these collaterals become critically compromised by an obstruction, then the collateral circulation is jeopardized, and the coronary perfusion pressure is effectively reduced to ischemic levels. Any stress with the effect of either increasing myocardial oxygen utilization or decreasing oxygen delivery, e.g., atrial pacing, 


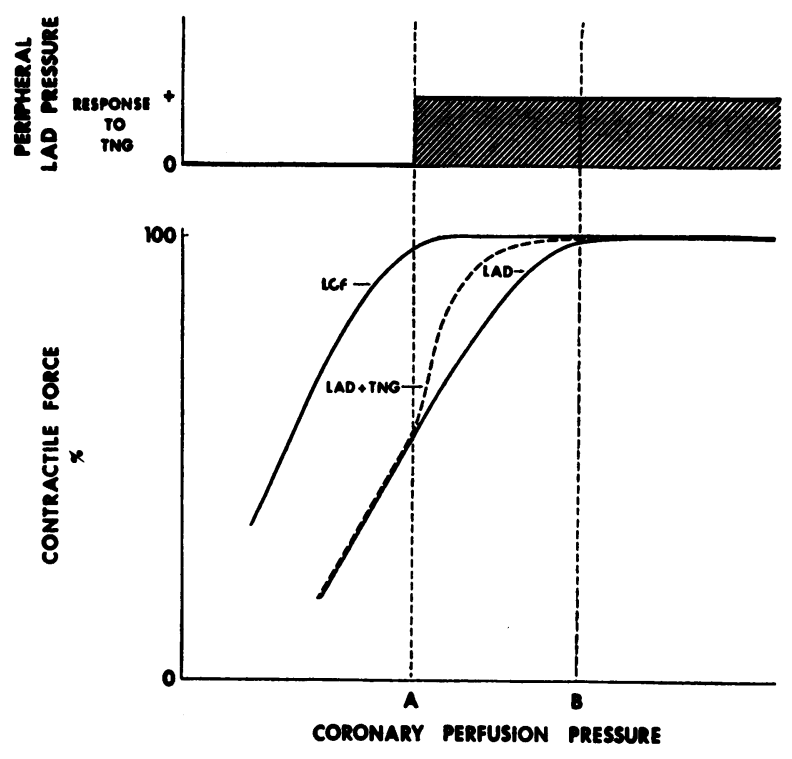

Figure 7 Diagrammatic presentation of the effect of TNG on peripheral LAD pressure and LAD and LCf contractile force at varying levels of coronary perfusion pressure. This graph is based on data as described in the text and presented in Table I, but is itself not a summary of the actua experimental points.

positive inotropic agents, hypoxia, decreased red cell mass, would also be expected to yield similar results.

Indices of collateral function most frequently used have been peripheral coronary pressure $(14,21-24)$, retrograde flow $(14,21,23-26)$, radioisotopic determination of collateral flow $(15,23,24,27-30)$, and microsphere determination of flow distribution (31). However, cer-

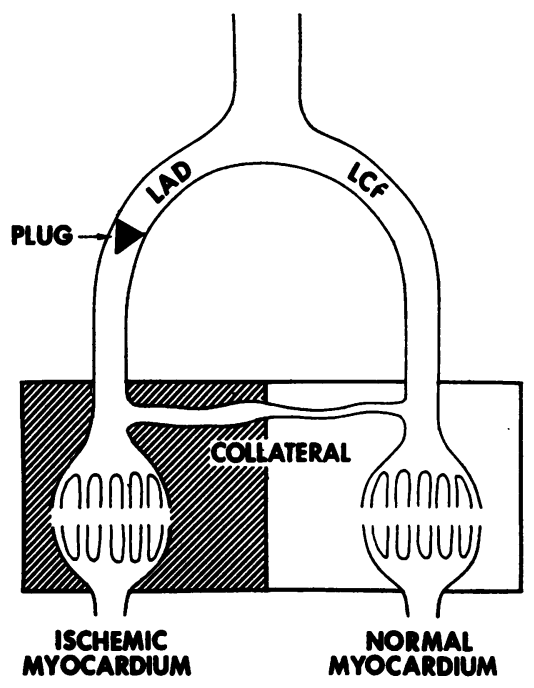

Figure 8 Model of the coronary circulation with an occlusive plug in the LAD. The coronary arteriolar vascular bed contains resistance vessels that dilate briefly in response to TNG. These vessels regain their tone before the response of coronary collaterals detailed in Fig. 9 is fully evident.

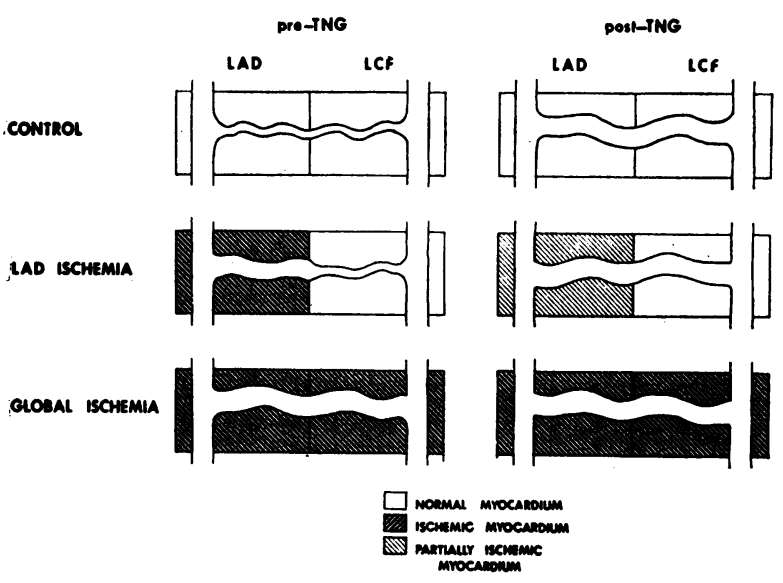

Figure 9 Detailed model of collateral vascular bed before (pre-TNG) and after (post-TNG) intracoronary nitroglycerin administration under various conditions of myocardial perfusion. Changes in shading indicate changing myocardial ischemia. See text.

tain of these indices are incapable of monitoring changes in myocardial blood flow, and none is able to measure the effect of changes in collateral flow on myocardial function. Recent work (32) indicates that the technique of retrograde flow used by Fam and McGregor (14) does not accurately estimate myocardial blood flow in an area supplied by collaterals, and furthermore in some cases may not indicate directional changes in collateral flow. An index assessing the functional effects of changes in collateral flow is desirable, and the response of isometric strain gauge arches is such an index. Although their aims and interpretations were different, Wiggers and Green (33) in 1936 suggested only direct myographic recordings should be used to assess changes in flow to ischemic myocardium. Other investigators have employed strain gauge arches (34), and work done in this laboratory" has demonstrated the sensitivity of strain gauge arch recordings in ischemic situations.

Although most investigators have found that TNG does not improve indices of collateral function after acute coronary occlusion $(14,21,23,35)$, several recent communications have found conflicting results (36, 37). Our data show no change in distal LAD pressure after TNG administration in this situation. Because of the experimental protocol it was not possible to assess the response of the strain gauge arch, which recorded either very minimal systolic shortening or even systolic bulging in the area supplied by the recently occluded LAD. With a modified experimental protocol permitting separate LAD and LCf perfusion, collateral function

'Downey, J. M., E. S. Kirk, D. F. Cowan, E. H. Sonnenblick, and C. W. Urschel. The adequacy of coronary perfusion during acute hemorrhagic hypotension. Submitted for publication. 
could be demonstrated by reversing ischemia caused by subtotal occlusion of the LAD with increased LCf perfusion. However, intracoronary TNG administered under similar circumstances was unable to alter the degree of ischemia. ${ }^{5}$

As noted by Schaper (38), immediately after coronary occlusion, coronary collaterals are vessels with $40-\mu$ diameters and sometimes only one cell layer of smooth muscle. Blum, Alpern, Jaffe, Lang, and Corday (39) concluded that dogs have even smaller functional intercoronary anastomoses after acute coronary ligation. Several days after coronary occlusion with Ameroid constrictors (Tenneco Chemicals Inc., American Plastics Div., Bainbridge, N. Y.), mitotic activity in these vessels is maximal, and by $3-4 \mathrm{wk}$ there is a significant smooth muscle layer, albeit still somewhat disorganized, and an enlarged vessel diameter (38). For this study an interval of $2 \frac{1}{2}-4$ wk after occlusion of the LAD was chosen as a compromise. To insure responsiveness to TNG in developing collaterals, formation of a hyperplastic smooth muscle layer was necessary. To maximize the gradient across the obstruction and therefore the possibility of creating selective ischemia in the distribution of the occluded vessel, it was necessary to restudy the animals as soon as possible. In a small series of animals studied at 10-12 wk, however, qualitatively similar results were also noted. ${ }^{5}$

Segments of the large coronary artery proximal to the collateral vessels cannot account for the observed responses to TNG. Winbury, Howe, and Hefner (2) and Fam and McGregor (1) have noted that large and small coronary arteries can be differentiated by their response to ischemia and drugs such as TNG. Only the small arterioles autoregulate while the tone in large vessels is unaffected by changes in perfusion. Small vessels dilate immediately in response to $\mathrm{TNG}$ and then rapidly regain their normal tone whereas large vessels dilate slowly and maintain this lower resistance for 7-12 min. Large coronary arteries maintain their ability to dilate in response to TNG under ischemic conditions (2). In our model the absence of any salutary effects of TNG during global ischemia, when the large vessels are known to be responsive, precludes them as significant factors. Therefore, collateral vessels must account for the changes. The collaterals appear to have properties of both large and small vessels, i.e., they have the slow prolonged response of large vessels to TNG at normal perfusion pressures, but like small vessels become unresponsive to TNG at decreased pressure. Thus our findings suggest that the collateral may be a hybrid vessel.

The clinical efficacy of TNG for the relief of angina pectoris is unquestioned. Pharmacologic agents as gang-

\footnotetext{
${ }^{5}$ Unpublished observations of the authors.
}

lionic blockers (40), which are similar to TNG in their effects on the peripheral vasculature, and other coronary vasodilators such as dipyridamole (41) are clinically ineffective in the anginal syndrome. Furthermore, TNG may be effective without eliciting falls in blood pressure or other signs of cardiac unloading (42). Thus an additional property of TNG that accounts for some of its salutary clinical effects may be its ability to dilate collaterals. It is to be noted, however, that we administered TNG directly into the coronary circulation. Only during experimental studies is this realized in man (5), and therefore the special conditions of our protocol must not be overlooked. Collateral vessels in the dog heart are often epicardial, whereas these vessels tend to be intramural in man (38). This anatomic difference might be cause to caution one further against directly applying our conclusions to the clinical situation. However, a recent intraoperative study in man (19) has demonstrated a dilatory effect of TNG on collateral vessels analogous to our experimental observations.

Forman, Kirk, Downey, and Sonnenblick (18) as well as others $(5,43)$ have demonstrated the transient increase in coronary blood flow lasting less than 1-2 min after the intracoronary administration of TNG. This interval is significantly shorter than the duration of collateral dilatation demonstrated in this study (range $2 \frac{1}{2}-8$ min; average $5 \mathrm{~min}$ ). McGregor and Fam (44) postulated that unless there were a differential effect of dilators on collaterals and resistance vessels, these agents might induce a coronary steal and thus further deprive the ischemic area of blood flow. Recently TNG has been shown to cause a coronary steal (18). Therefore coronary vasodilatation alone could be deleterious by initiating a steal. However, TNG's ability to dilate coronary collaterals for significantly longer intervals than the normal resistance vessels provides a unique way of furnishing increased collateral flow to nutritionally deprived myocardium.

The mode of action of TNG may depend on the pathological circumstance in which it is used. Thus, with prior major but incomplete obstruction of a large coronary artery, collaterals may be established. With subsequent completion of this obstruction and ensuing myocardial infarction, the response of the remaining collaterals to TNG may be beneficial. In the absence of prior collaterals or in situations where all major vessels are equally diseased, the effectiveness of TNG may reflect its ability to reduce venous return and systolic loading of the heart. Such differences may also exist in the setting of angina pectoris.

\section{ACKNOWLEDGMENTS}

This study was supported by USPHS Grants HE-110306 and HE-14561 and Training Grants 5-TO1-HE-05679 and 5-TO1-HE-05890. 


\section{REFERENCES}

1. Fam, W. M., and M. McGregor. 1968. Effect of nitroglycerin and dipyridamole on regional coronary resistance. Circ. Res. 22: 649.

2. Winbury, M. M., B. B. Howe, and M. A. Hefner. 1969. Effect of nitrates and other coronary dilators on large and small coronary vessels : an hypothesis for the mechanism of action of nitrates. J. Pharmacol. Exp. Ther. 168: 70 .

3. Brachfeld, N., J. Bozer, and R. Gorlin. 1959. Action of nitroglycerin on the coronary circulation in normal and in mild cardiac subjects. Circulation. 19: 697.

4. Cowan, C., P. V. M. Duran, G. Corsini, N. Goldschlager, and R. J. Bing. 1969. The effects of nitroglycerin on myocardial blood flow in man: measured by coincidence counting and bolus injections of ${ }^{84}$ rubidium. Am. J. Cardiol. 24: 154.

5. Ganz, W., and H. S. Marcus. 1972. Failure of intracoronary nitroglycerin to alleviate pacing-induced angina. Circulation. 46: 880.

6. Schnaar, R. L., and H. V. Sparks. 1972. Response of large and small coronary arteries to nitroglycerin, $\mathrm{NaNO}_{2}$, and adenosine. Am. J. Physiol. 223: 223.

7. Gensini, G. G., A. E. Kelly, B. C. B. DaCosta, and P. P. Huntington. 1971. Quantitative angiography: the measurement of coronary vasomobility in the intact animal and man. Chest. 60: 522 .

8. Mason, D. T., and E. Braunwald. 1965. The effects of nitroglycerin and amyl nitrite on arteriolar and venous tone in the human forearm. Circulation. 32: 755.

9. Mason, D. T., R. Zelis, and E. A. Amsterdam. 1971. Actions of the nitrites on the peripheral circulation and myocardial oxygen consumption: significance in the relief of angina pectoris. Chest. 59: 296.

10. Carson, R. P., W. S. Wilson, M. J. Nemiroff, and W. J. Weber. 1969. The effects of sublingual nitroglycerin on myocardial blood flow in patients with coronary artery disease or myocardial hypertrophy. Am. Heart J. 77: 579 .

11. Marchetti, G. V., L. Merlo, and R. M. Antognetti. 1964. The effects of nitroglycerin on the coronary blood flow and oxygen consumption of the myocardium in anesthetized dogs. Am. J. Cardiol. 13: 51 .

12. Rees, J. R., V. J. Redding, R. Ashfield, D. Gibson, and C. J. Gavey. 1966. Myocardial blood flow measurement with ${ }^{133}$ xenon: effect of glyceryl trinitrate in dogs. $\mathrm{Br}$. Heart J. 28: 374

13. Vyden, J. K., M. Carvalho, E. Boszormenyi, T.-W. Lang, H. Bernstein, and E. Corday. 1970. Effect of glyceryl trinitrate (nitroglycerin) on the systemic and coronary circulation of the dog. Am. J. Cardiol. 25: 53 .

14. Fam, W. M., and M. McGregor. 1964. Effect of coronary vasodilator drugs on retrograde flow in areas of chronic myocardial ischemia. Circ. Res. 15: 355.

15. Horwitz, L. D., R. Gorlin, W. J. Taylor, and H. G. Kemp. 1971. Effects of nitroglycerin on regional myocardial blood flow in coronary artery disease. J. Clin. Invest. 50: 1578 .

16. Cohen, M. V., and P. Eldh. 1973. Experimental myocardial infarction in the closed-chest dog: controlled production of large or small areas of necrosis. $\mathrm{Am}$. Heart J. In press.

17. Mosher, P., J. Ross, Jr., P. A. McFate, and R. F. Shaw. 1964. Control of coronary blood flow by an autoregulatory mechanism. Circ. Res. 14: 250.

18. Forman, R., E. S. Kirk, J. M. Downey, and E. H.
Sonnenblick. 1973. Nitroglycerin and heterogeneity of myocardial blood flow: reduced subendocardial blood flow and ventricular contractile force, $J$. Clin. Invest. 52: 905 .

19. Goldstein, R. E., E. B. Stinson, and S. E. Epstein. 1973. Effects of nitroglycerin on coronary collateral function in patients with coronary occlusive disease. Am. J. Cardiol. 31: 135 (Abstr.)

20. Berne, R. M., R. Rubio, J. G. Dobson, Jr., and R. R. Curnish. 1971. Adenosine and adenine nucleotides as possible mediators of cardiac and skeletal muscle blood flow regulation. Circ. Res. Suppl. 28: 115.

21. Kattus, A. A., and D. E. Gregg. 1959. Some determinants of coronary collateral blood flow in the openchest dog. Circ. Res. 7: 628.

22. Grayson, J., and M. Irvine. 1968. Myocardial infarction in the monkey: studies on the collateral circulation after acute coronary occlusion. Cardiovasc. Res. 2: 170.

23. Pasyk, S., C. M. Bloor, E. M. Khouri, and D. E. Gregg. 1971. Systemic and coronary effects of coronary artery occlusion in the unanesthetized dog. $\mathrm{Am} . J$. Physiol. 220: 646 .

24. Elliot, E. C., C. M. Bloor, E. L. Jones, W. J. Mitchell, and D. E. Gregg. 1971. Effect of controlled coronary occlusion on collateral circulation in conscious dogs. Am. J. Physiol. 220: 857.

25. Gundel, W. D., B. G. Brown, and V. L. Gott. 1970. Coronary collateral flow studies during variable aortic root pressure waveforms. J. Appl. Physiol. 29: 579.

26. Menick, F. J., F. C. White, and C. M. Bloor. 1971. Coronary collateral circulation: determination of an anatomical anastomotic index of functional collateral flow capacity. Am. Heart J. 82: 503.

27. Hollander, W., I. M. Madoff, and A. V. Chobanian. 1963. Local myocardial blood flow as indicated by the disappearance of $\mathrm{NaI}^{131}$ from the heart muscle: studies at rest, during exercise, and following nitrite administration. J. Pharmacol. Exp. Ther. 139: 53.

28. Linder, E., and T. Seeman. 1967. Effects of Persantin and nitroglycerin on myocardial blood flow during temporary coronary occlusions in dogs Angiologica. 4: 225.

29. Haft, J. I., and A. N. Damato. 1969. Measurement of collateral blood flow after myocardial infarction in the closed-chest dog. Am. Heart J. 77: 641.

30. Rees, J. R., and V. J. Redding. 1969. Increase in myocardial collateral capacity following drug-induced coronary vasodilatation: a preliminary report. Am. Heart J. 78: 224.

31. Becker, L. C., and B. Pitt. 1971. Collateral blood flow in conscious dogs with chronic coronary artery occlusion. Am. J. Physiol. 221 : 1507.

32. Cibulski, A. A., P. H. Lehan, and H. H. Timmis. 1972. Retrograde flow technique vs. krypton-85 clearance technique for estimation of myocardial collaterals. $\mathrm{Am}$. J. Physiol. 223: 1081.

33. Wiggers, C. J., and H. D. Green. 1936. The ineffectiveness of drugs upon collateral flow after experimental coronary occlusion in dogs. Am. Heart J. 11: 527.

34. Nishimura, A., F. Giron, W. C. Birtwell, and H. S. Soroff. 1970. Evaluation of collateral blood supply by direct measurement of the performance of ischemic myocardial muscle. Trans. Am. Soc. Artif. Intern. Organs. $16: 450$.

35. Becker, L. C., N. J. Fortuin, and B. Pitt. 1971. Effect of ischemia and antianginal drugs on the distribution of 
radioactive microspheres in the canine left ventricle. Circ. Res. 28: 263.

36. Redding, V. J.. and J. R. Rees. 1968. Early changes in collateral flow following coronary artery ligation: the role of the sympathetic nervous system. Cardiovasc. Res. $3: 219$.

37. Brown, B. G., W. D. Gundel, V. L. Gott, and J. W. Covell. 1972. Hemodynamic determinants of retrograde arterial coronary flow following acute coronary occlusion. Circulation. 46(Suppl. 2) : 100 (Abstr.)

38. Schaper, W. 1971. The Collateral Circulation of the Heart. North-Holland Publishing Co., Amsterdam.

39. Blum, R. L., H. Alpern, H. Jaffe, T.-W. Lang, and E. Corday. 1970. Determination of interarterial coronary anastomosis by radioactive spherules. Effect of coronary occlusion and hypoxemia. Am. Heart J. 79: 244.
40. Winbury, M. M. 1964. Experimental approaches to the development of antianginal drugs. Adv. Pharmacol. $3: 1$.

41. McGregor, M. 1966. Drugs for the treatment of angina pectoris. In International Encyclopedia of Pharmacology and Therapeutics. Clinical Pharmacology. L. Lasagna, editor. Pergamon, Oxford. II (Sect. 6) : 377.

42. Hilger, H. H. 1969. Experimentelle Prüfung der Wirkung von Coronardilatatoren am Menschen. NaunynSchmiedebergs Arch. Pharmakol. Exp. Pathol. 263: 168.

43. Bernstein, L., G. C. Friesinger, P. R. Lichtlen, and R. S. Ross. 1966. The effect of nitroglycerin on the systemic and coronary circulation in man and dogs: myocardial blood flow measured with xenon ${ }^{133}$. Circulation. 33: 107.

44. McGregor, M, and W. M. Fam. 1966. Regulation of coronary blood flow. Bull. N. Y. Acud. Med. 42: 940. 